\title{
A FORMAÇÃO INTERDISCIPLINAR DE LICENCIANDOS EM CIÊNCIAS DA NATUREZA E O APRENDIZADO DAS CIÊNCIAS DO CÉU
}

\author{
Gisele Soares Lemos Shaw ${ }^{1}$ \\ João Batista Teixeira da Rocha²
}

\begin{abstract}
RESUMO
A interdisciplinaridade tem sido incentivada no ensino de ciências, mas sua operacionalidade precisa ser mais bem investigada. Estudos têm sido desenvolvidos para compreender a formação interdisciplinar no Ensino Superior, uma vez que essa formação pode ser base para que professores, ou futuros professores, desenvolvam práticas interdisciplinares na escola. Baseado nos estudos de Spelt et al. (2009) acerca da constituição do pensamento interdisciplinar em estudantes de Ensino Superior, foi investigado o processo de formação interdisciplinar de três licenciandos em Ciências da Natureza durante uma oficina de Astronomia desenvolvida com estudantes do Ensino Fundamental. Por meio da análise textual discursiva de dados provindos de questionário, formulário de planejamento da oficina, projeto da oficina, planos de aula, anotações sobre a apresentação verbal da experiência, relato da experiência em formato de artigo, formulário autoavaliativo e entrevista, foram analisados conhecimentos e habilidades interdisciplinares desenvolvidos por esses licenciandos. Foi observada a aquisição de alguns conhecimentos e habilidades pelos participantes, mas a ausência de outros indicou que o pensamento interdisciplinar ainda não havia sido adquirido na experiência, mas estava em processo de formação.
\end{abstract}

Palavras-chave: Ensino Superior. Formação interdisciplinar. Interdisciplinaridade.

\section{THE INTERDISCIPLINARY TRAINING OF UNDERGRADUATES IN NATURAL SCIENCES} AND THE LEARNING OF THE HEAVEN SCIENCE

\begin{abstract}
Interdisciplinary has been encouraged in science education, but we must investigate how to do it. Studies in development seek to understand interdisciplinary training in higher education, since such training may be the basis for teachers or future teachers developed interdisciplinary practices in school. Based studies by Spelt et al (2009) on the constitution of interdisciplinary thinking in higher education students, the process of interdisciplinary formation of three licentiates in the Natural Sciences, we investigated during an astronomy workshop developed with elementary students. Through the discursive textual analysis of data from questionnaire, workshop-planning form, workshop project, lesson plans, notes on a verbal presentation of the experience, report of the experience in an paper format, self-evaluation form and interview were analyzed knowledge and interdisciplinary skills developed by these undergraduates. Participants observed an acquisition of some knowledge and skills, but the absence of others indicated that the graduates did not acquire interdisciplinary thinking in the experience, but it was in the process of formation.
\end{abstract}

Keywords: Higher Education. Interdisciplinary Training. Interdisciplinary.

Recebido em: $15 / 5 / 2018$

Aceito em: $23 / 8 / 2018$

\footnotetext{
$\overline{{ }^{1} \text { Professora-adjunta }}$ da Universidade Federal do Vale do São Francisco - Univasf. Doutoranda em Educação em Ciências: Química da Vida e da Saúde pela Universidade Federal do Rio Grande do Sul (UFRGS). Mestre em Ensino, Filosofia e História das Ciências pela Universidade Federal da Bahia (Ufba) e Universidade Estadual de Feira de Santana (Uefs). Especialista em A Construção do Conhecimento e o Ensino de Ciências e Licenciada em Pedagogia pela Uesb (2004). Possui experiência em educação, com ênfase em Ensino de Ciências, atuando principalmente nos seguintes temas: Cultura Escolar, Didática, Ensino de Ciências, Interdisciplinaridade e História da Disciplina Escolar Ciências. giseleshaw@hotmail.com

${ }^{2}$ Graduação em Ciências Biológicas (1986) e Doutorado em Ciências Biológicas (Bioquímica) (1996) pela Universidade Federal do Rio Grande do Sul - UFRGS. Pós-Doutorado na UFRJ - Departamento de Bioquímica Médica no Laboratório de Bioenergética (1997-1998). Trabalha na área de bioquímica, toxicologia e farmacologia de organocalcogênio, papel do estresse oxidativo em patologias humanas e experimentais e educação em ciências. Têm experiências na área de toxicologia, sistema nervoso central (incluindo psicofarmacologia e desenvolvimento), farmacologia de organocalcogênios e interação entre calcogênios e tióis. Participa de atividade relacionados ao ensino de ciências e como melhorar o ensino de ciências por meio da interação entre cientistas e estudantes e professores do Ensino Médio e fundamental. jbtrocha@yahoo.com.br
} 
Apesar de a interdisciplinaridade no ensino ser fomentada desde a década de 70 (FAZENDA, 2010, 2012a, 2012b) e estar prevista nos Parâmetros Curriculares Nacionais - PCNs (BRASIL, 1998) -, ainda há necessidade de alargamento das práticas relativas à mesma. Esse imperativo de aprofundamento também ocorre em âmbito internacional, pois, conforme Fazenda (2010), uma formação interdisciplinar sólida tem sido investigada por diversos pesquisadores provindos de outros países, tais como Julie Klein, da Wayne State University e Willian Newell, da Miami University, e provém de "prática em situação real e contextualizada" (FAZENDA, 2010, p. 176).

Além disso, ainda segundo Fazenda (2010), a formação à interdisciplinaridade, pela interdisciplinaridade e para a interdisciplinaridade exige "um alto grau de amadurecimento intelectual e prático, uma aquisição no processo reflexivo que vai além do simples nível de abstração" (p. 175-176). Esse nível de formação propicia uma nova atitude diante do conhecimento - uma atitude interdisciplinar, construída por meio de uma sólida formação interdisciplinar (FAZENDA, 2010). Como não existe uma Teoria Geral da Interdisciplinaridade (KLEIN, 2012), ou um modelo de prática interdisciplinar a ser seguido, é preciso que os professores construam seu percurso formativo por meio da habilidade de refletir na prática e sobre a prática, o que Shön trata como reflexão na ação e reflexão sobre a ação (SHÖN, 2000), além de desenvolver habilidades de refletir acerca de seu próprio pensamento, que Ivanitskaya et al. (2002) tratam como habilidades metacognitivas. Para Klein (2012): “(...) os professores precisam de uma epistemologia da prática, marcada pela união reflexiva entre o pensar e o fazer. Nessas condições, a capacidade interdisciplinar não é periférica, mas central" (KLEIN, 2012, p. 131). De acordo com Fazenda (2012a), um projeto interdisciplinar requer "a formação de um professor/pesquisador, daquele que busque a redefinição contínua de sua práxis" (p. 51).

Assim, diante da necessidade de aprofundar os estudos sobre a formação interdisciplinar de estudantes do Ensino Superior e de identificar habilidades e conhecimentos necessários a essa formação, foram investigados três licenciandos em Ciências da Natureza no decorrer de sua participação em atividade de uma disciplina universitária. Esses estudantes foram desafiados a refletir acerca de sua prática e da aprendizagem de seus alunos no âmbito de uma oficina pedagógica potencialmente interdisciplinar, construída pelos mesmos. Essa oficina - $O$ aprendizado das ciências do céu por meio do Sistema solar - foi desenvolvida com estudantes do sexto ano do Ensino Fundamental de uma escola da rede privada de ensino e buscou propiciar o aprendizado de conteúdos de Astronomia por esses alunos, por meio da articulação interdisciplinar.

Utilizando como base norteadora as ideias de Spelt et al. (2009) acerca da constituição do pensamento interdisciplinar, objetivou-se identificar nesses futuros professores de ciências o desenvolvimento de conhecimentos e habilidades interdisciplinares no decorrer da experiência. Os dados foram coletados mediante a análise de questionário, formulário de planejamento da oficina, projeto da oficina, planos de aula, anotações sobre a apresentação verbal da experiência, relato da experiência em formato de artigo, 
formulário autoavaliativo e entrevista com um dos três participantes da pesquisa. ${ }^{3}$ Esses dados foram analisados mediante análise textual discursiva (MORAES, 2003; MORAES; GALIAZZI, 2006).

\section{O ENSINO DE ASTRONOMIA NA LITERATURA RECENTE}

Apesar de o ensino de Astronomia estar previsto na legislação educacional brasileira vigente, ainda há carências, tanto no ensino quanto na pesquisa. Mesmo que os conteúdos de Astronomia sejam caracterizados como motivadores aos estudantes (BARROS; ASSIS; LANGHI, 2016; MIRANDA et al., 2016) e potencialmente interdisciplinares (BARROS; ASSIS; LANGHI, 2016; CARVALHO, et al., 2016; LANGHI; NARDI, 2014), apontam-se dificuldades quanto à operacionalização desse ensino nas escolas, que perpassam desde problemas de falta de preparação dos professores (CARVALHO et al., 2016) até a carência de materiais didáticos que tragam informações adequadas do assunto (CARVALHO et al., 2016; MIRANDA et al., 2016). Alguns trabalhos recentes discutem a importância do ensino de Astronomia, trazendo estratégias didáticas de como desenvolvê-lo na escola, além das dificuldades encontradas, como será visto adiante.

Pesquisando o que tem sido publicado sobre o ensino de Astronomia, Langhi e Nardi (2014) fizeram uma pesquisa bibliográfica acerca da Educação em Astronomia, realizando a análise dos dados a partir do Discurso do Sujeito Coletivo. Para fazer o recorte, selecionaram artigos publicados entre os anos de 2004 e 2014 em revistas da área de ensino de Qualis A1 e A2, incluindo a Revista Latino-Americana de Educação em Astronomia - Relea -, a única nacional destinada a discutir a educação em Astronomia. Dos 138 artigos encontrados, apontou-se que a Revista Brasileira de Ensino de Física possui $65 \%$ dos artigos produzidos sobre o ensino de astronomia no período pesquisado. Foi indicado, também, que, a partir de 2009, houve aumento no número de publicações, considerando a amostra pesquisada. Dentre as ideias centrais encontradas por Langhi e Nardi (2014) nos discursos dos pesquisadores, destaca-se o caráter interdisciplinar da Astronomia, atribuído à possibilidade de realizar interfaces entre disciplinas. Apesar das vantagens trazidas pelo ensino de Astronomia, os mesmos autores apontaram o descaso quanto à abordagem desse tema na educação brasileira e indicaram a importância de serem realizadas mais pesquisas que focalizem nos conteúdos a serem ensinados e nos modos de ensinar astronomia.

Considerando as potencialidades do ensino de Astronomia, Carvalho et al. (2016) discutiram acerca do interesse e do contato de 341 estudantes do Ensino Médio de uma escola estadual no município de São João Del-Rei/MG, com temas de astronomia. Para isso, eles desenvolveram uma atividade expositiva e utilizaram o programa Strellarium com estudantes. Assim como Langhi e Nardi (2014), Carvalho et al. (2016) também assinalaram que uma das vantagens da Astronomia é seu caráter potencialmente interdisciplinar, pois se relaciona com conteúdos de diversas disciplinas: Matemática, Química,

${ }^{3}$ Entrevistamos apenas um dos participantes porque os outros dois não se disponibilizaram a isso. 
Biologia, História, Geografia e Filosofia. Esses autores ainda apontaram o uso de simuladores computacionais como boas ferramentas de ensino e aprendizagem, como é o caso do software Strellarium, usado para a exploração do ambiente celeste.

Carvalho et al. (2016) perguntaram aos estudantes, por meio de questionário aplicado antes e depois da intervenção na escola, se estes já haviam tido contato com a Astronomia, e se eles se sentiam atraídos por esse tema. A maioria dos estudantes afirmou não ter tido contato com a Astronomia. Dos estudantes que se aproximaram do tema, a maior parte deles assegurou que isso se deu por meio de filmes, atividades escolares ou documentários. A maioria dos estudantes também indicou ter curiosidade pela Astronomia e já ter visto um documentário ou lido algum livro relacionado, apesar de quase um terço da amostra afirmar não se motivar pelo assunto. Após a realização da intervenção realizada, foi observado que a maior parte dos alunos mostrou que a atividade contribuiu para aumentar seu fascínio pela Astronomia. Houve redução em $15 \%$ do número de discentes que indicou não ter entusiasmo pelo tema. Os autores apontam a necessidade de mais investigações que esclareçam acerca dos interesses específicos dos alunos em assuntos da Astronomia.

Investigando a potencialidade de jogos didáticos no ensino de Astronomia, Miranda et al. (2016) relataram a aplicação de três desses jogos com estudantes do Ensino Fundamental de uma escola pública do Estado do Rio de Janeiro. Essa atividade, ligada ao trabalho do Pibid ${ }^{4}$ Ciências Naturais - Pádua (da Universidade Federal Fluminense) -, teve como objetivo preparar esses estudantes para a realização da prova da Olimpíada Brasileira de Astronomia (OBA). Os jogos envolvidos foram "Responde ou Passa - Astronomia", "Jogos dos Astros" e "Sorte ou Azar na Astronomia", que abordaram os temas "Origem do Universo", "Corpos Celestes", "Pontos Cardeais", "Estações do Ano", "Solstício e Equinócio", "Movimentos da Terra", "Fases da Lua" e "Eclipses".

Sobre a confecção dos jogos, Miranda et al. (2016) informaram que estes “(...) foram produzidos com materiais de baixo custo e fácil aquisição, além do uso de computador, impressora e plastificadora, usados para padronização e aumento da durabilidade do jogo" (MIRANDA et al., 2016, p. 4). Além disso, para avaliar a usabilidade, compreensão e clareza dos estudantes quanto aos jogos, foi aplicado um questionário contendo nove questões, sendo oito delas fechadas e uma aberta.

De acordo com os dados analisados, Miranda et al. (2016) apontaram que a maioria dos participantes aprovou a utilização dos jogos: indicou a clareza e a objetividade das perguntas, achou o visual agradável, afirmou se sentir estimulada com a utilização dos jogos e certificou que os jogos abordaram conteúdos tratados em sala de aula. Segundo os mesmos autores, o jogo que teve melhor aceitação foi o Sorte ou Azar na Astronomia. Eles pontuaram que, após a atuação do Pibid com estudantes, houve au-

\footnotetext{
${ }^{4}$ Pibid - Programa Institucional de Bolsas de Iniciação à Docência. Programa do Ministério da Educação do Brasil voltado ao estímulo à docência em escolas por estudantes de cursos de Licenciatura por meio da disponibilização de bolsas e das parcerias entre instituições de Ensino Superior e instituições escolares. Mais informações disponíveis em: http://portal.mec.gov.br/pibid. Acesso em: 14 maio 2018.
} 
mento no número de alunos participantes do $\mathrm{OBA},{ }^{5}$ além do crescimento no número de professores motivados a colaborar na preparação dos estudantes para essas provas e também no aumento da quantidade de medalhas recebidas.

Pesquisando acerca do uso de Novas Tecnologias no ensino de Astronomia, Sampaio e Rodrigues (2015) apresentaram o impacto do uso do software de simulação astronômica Strellarium na aquisição de conhecimentos de Astronomia por 150 estudantes de escolas públicas participantes. Os autores aplicaram questionários antes e depois do desenvolvimento de atividades, envolvendo aula expositiva e utilização do Strellarium. Segundo Sampaio e Rodrigues (2015), a maioria dos estudantes declarou não conhecer o software e também nunca ter estudado sobre Astronomia, mas demonstrou interesse no programa. Eles também aferiram uma integração positiva entre os estudantes e os conteúdos de Astronomia com as atividades realizadas.

Santana (2015) investigou a utilização da abordagem Ciência Tecnologia Sociedade (CTS) na formação continuada de professores para o ensino de Astronomia, por intermédio de um curso de formação continuada ofertado. Esse curso foi desenvolvido por meio da situação problema "Centro de Lançamento de Alcântara", e foi pautado na perspectiva de ensinar e aprender através da reflexão na ação. A pesquisa desenvolvida por Santana (2015) foi qualitativa, do tipo Pesquisação, tendo como participantes sete professores das redes federal, estadual e municipal de ensino do Estado do Pará. Seu curso teve duração de 80 horas e foi organizado em torno da dinâmica dos três momentos pedagógicos de Delizoicov, Angotti e Pernambuco (2002), composto pelas seguintes etapas: problematização inicial, organização do conhecimento e aplicação do conhecimento.

Para a escrita de sua dissertação de Mestrado, Santana (2015) analisou uma das atividades do curso, que envolveu o problema da construção da Estação de Lançamento de Alcântara. A partir dos dados coletados por meio de diversas fontes produzidas durante a investigação, tais como diário de formação, relatos de experiência, entrevistas semiestruturadas, rodas de conversa, além da gravação de discussões entre os participantes, a autora analisou o caso da Estação Alcântara por meio de dois vieses: a localização da construção e a questão dos quilombolas que foram retirados do local. A mesma autora verificou a importância da atividade formativa que contribuiu para agregar valor: a sua visão de interdisciplinaridade, contextualização e autonomia; a atitudes de reflexão na ação; a conhecimentos sobre as atividades CTS; à motivação e ao trabalho coletivo. Ela também concluiu que a abordagem CTS no ensino de Astronomia contribui para a formação crítico-reflexiva e autônoma de professores. A mesma ainda mostrou que os docentes participantes da investigação indicaram que a interdisciplinaridade, como categoria de ação, favorece uma prática diferenciada, além da cooperação no grupo.

${ }_{5}^{5}$ Olimpíada Brasileira de Astronomia (OBA). Disponível em: http://www.oba.org.br/site/. Acesso em: 14 maio 2018. 
Considerando as pesquisas recentes apresentadas, foi observado que ainda há muito o que ser investigado no ensino de Astronomia. Além disso, é importante que essas investigações alcancem as escolas por meio da qualificação dos professores para o ensino desse campo do saber, que tem demonstrado ser motivador e facilitador da prática interdisciplinar.

\section{A FORMAÇÃO DO PENSAMENTO INTERDISCIPLINAR}

Apesar de a interdisciplinaridade no ensino ser estudada desde a metade do século 20, ainda não existe um consenso sobre o conceito de interdisciplinaridade ou um modelo de ensino que propicie esse fenômeno. Do ponto de vista epistemológico, porém, Lenoir (2005) aponta três perspectivas da interdisciplinaridade: a perspectiva europeia, a estadunidense e a brasileira. Segundo o autor, essas lógicas são complementares. Logo, a interdisciplinaridade precisa ser compreendida mediante as três dimensões: a lógica do sentido, a lógica da funcionalidade e a lógica da intencionalidade fenomenológica.

A interdisciplinaridade pelo viés europeu, segundo Lenoir (2005), segue a lógica do sentido, logo é mais conceitual, abrangendo aspectos teóricos. Nessa abordagem, há a predominância da preocupação com a reflexão epistemológica sobre os saberes disciplinares em interação. Nessa visão, a interdisciplinaridade no ensino é necessária para que as pessoas aprendam a compreender a complexidade do conhecimento e a relação entre saberes.

A interdisciplinaridade, sob a visão estadunidense, é mais pragmática, vista como um fenômeno necessário à resolução de problemas sociais. Essa abordagem é classificada, para Lenoir (2005), segundo a lógica da funcionalidade. O exercício da interdisciplinaridade é visto como algo instrumental, funcional à sociedade. Nessa perspectiva, a interdisciplinaridade no ensino objetiva preparar os estudantes à resolução de problemas reais.

Na perspectiva brasileira, a interdisciplinaridade é compreendida principalmente por meio dos estudos de Ivani Fazenda (2010, 2012a, 2012b). Ela é entendida como um processo que depende do sujeito, que deve desenvolver uma atitude interdisciplinar diante do conhecimento. Essa abordagem é classificada para Lenoir (2005) como a perspectiva da intencionalidade fenomenológica. Nesse caso, apenas por meio de uma atitude interdisciplinar do sujeito é possível lidar com a complexidade do conhecimento. Assim, a interdisciplinaridade no ensino depende da atitude interdisciplinar docente, ainda que Ivani Fazenda reconheça a importância do trabalho coletivo para a realização de práticas interdisciplinares.

Compreende-se que a interdisciplinaridade seja um fenômeno necessário para lidar com a complexidade do conhecimento e que, para isso, é preciso que haja uma atitude interdisciplinar, pois esse movimento depende da vontade e do comprometimento de cada sujeito envolvido no processo. Mais ainda, entende-se que a realização da interdisciplinaridade no ensino depende do desenvolvimento de um pensamento interdisciplinar, que é fruto da mobilização de diversos conhecimentos e habilidades do sujeito, junto a condições apropriadas à prática interdisciplinar (SPELT et al., 2009). 
Logo, a perspectiva adotada neste artigo coaduna com Lenoir (2005) no que diz respeito à possibilidade de considerar as três lógicas da interdisciplinaridade como complementares, e também com as ideias de pensamento interdisciplinar trazidas por Spelt et al. (2009).

Spelt et al. (2009) investigaram a interdisciplinaridade no Ensino Superior e concluíram que é necessário que os estudantes desenvolvam um pensamento interdisciplinar. Segundo os autores, o pensamento interdisciplinar é resultante da aprendizagem no Ensino Superior interdisciplinar e é um tipo de pensamento complexo, constituído por um número de habilidades.

Spelt et al. (2009) explicaram o pensamento interdisciplinar por meio da teoria de Biggs (2003), e o trouxeram como resultado da interação entre o estudante (com seus conhecimentos e habilidades prévias) e um meio ambiente de aprendizagem propício, envolvendo o processo de aprendizagem. Então, para Spelt et al. (2009), para que o pensamento interdisciplinar seja desenvolvido no Ensino Superior é preciso que o estudante tenha certas características pessoais e experiências anteriores, além de que desenvolva determinados conhecimentos e habilidades que propiciem o processo. Também é preciso que o meio ambiente educativo seja voltado à interdisciplinaridade (por meio do currículo, professor, pedagogia e avaliação) e que seja desenvolvido um processo de aprendizagem dentro de padrões específicos e constituído por algumas atividades de aprendizagens propícias à interdisciplinaridade.

Spelt et al. (2009) apresentaram os elementos necessários à realização da interdisciplinaridade no ensino superior, que pode resultar no desenvolvimento do pensamento interdisciplinar. Conforme exposto, o pensamento interdisciplinar é composto pelos conhecimentos das disciplinas, dos paradigmas interdisciplinares e de conhecimentos acerca da própria interdisciplinaridade. Além disso, esse pensamento também é formado por habilidades de alta ordem cognitiva e por habilidades de comunicação. Inclusive, o estudante precisa ter características pessoais específicas para a prática da interdisciplinaridade, que são a curiosidade, o respeito, a abertura, a paciência, a diligência e a autorregulação, além de serem consideradas suas experiências anteriores, tanto sociais quanto educacionais (SPELT et al., 2009).

Fazenda (2010) trouxe alguns princípios cuja prática docente interdisciplinar precisa seguir, que são: humildade, coerência, respeito, espera e desapego. O respeito é a característica pessoal/princípio que converge entre Fazenda (2010) e Spelt et al. (2009). Newell e Green (1982) explicaram que estudantes de cursos interdisciplinares são mais abertos a novas ideias porque já se acostumaram a aprender com perspectivas diferentes, então eles se tornam pessoas mais tolerantes à incerteza do conhecimento e à ambiguidade.

O meio ambiente propiciador do pensamento interdisciplinar depende da existência de um currículo que tenha um equilíbrio entre a disciplinaridade e a interdisciplinaridade, cujo conhecimento disciplinar esteja dentro ou fora dos cursos interdisciplinares. Esse ambiente também depende de aspectos relacionados a seus professores: que haja uma comunidade intelectual focada na interdisciplinaridade, que tenha professores peritos ou experientes em interdisciplinaridade, que haja consenso sobre a interdisciplinaridade, além da existência de equipes de desenvolvimento e de ensino que 
desenvolvam e acompanhem esse currículo. O meio ambiente favorecedor da interdisciplinaridade também necessita de uma Pedagogia que busque o alcance da mesma, da aprendizagem ativa e da colaboração, além de um sistema de avaliação que considere a maturidade intelectual dos estudantes e que reconheça a importância da colaboração (SPELT et al., 2010).

Um processo de aprendizagem que favoreça o desenvolvimento do pensamento interdisciplinar depende de determinados padrões, que são: o avanço do processo de aprendizagem por fases e gradativo, a linearidade, a interatividade e ser marcado por questões encorajadoras. Além disso, esse processo de aprendizagem precisa ser desenvolvido com base em atividades que alcancem a interdisciplinaridade e que proporcionem a reflexão (SPELT et al., 2010). De acordo com Ivanitskaya et al. (2002), abordagens de ensino interdisciplinares são desenvolvidas junto ao amadurecimento ou desenvolvimento de habilidades de alta ordem cognitiva nos estudantes, tais como o pensamento crítico, habilidades metacognitivas e a epistemologia pessoal do indivíduo. Assim, essas abordagens contam com o aprimoramento cognitivo dos estudantes, em que a reflexão acerca do processo de ação e de pensamento é elemento preponderante.

Logo, observa-se o quão complexo é o pensamento interdisciplinar e que esse, conforme Spelt et al. (2009), não ocorre espontaneamente, mas depende de um processo longo de aprendizagem.

\section{CAMINHOS DA PESQUISA}

A compreensão da formação interdisciplinar de três licenciandos em Ciências da Natureza foi realizada a partir de uma investigação de natureza qualitativa. Os enunciados e ações desses participantes foram interpretados de modo a construir um entendimento dessa formação por meio da oficina potencialmente interdisciplinar que desenvolveram. De acordo com Moreira (2011), o interesse central da pesquisa qualitativa: “(...) está em uma interpretação dos significados atribuídos pelos sujeitos a suas ações em uma realidade socialmente construída, através de observação participativa, isto é, o pesquisador fica imerso no fenômeno de interesse" (p. 76). Ainda, além de a pesquisa ser qualitativa, os dados foram analisados sob um ponto de vista fenomenológico, a partir do viés subjetivo dessa experiência, sob o ponto de vista dos participantes.

$O$ universo da pesquisa foi a oficina pedagógica denominada $O$ aprendizado das ciências do céu por meio do sistema solar. Essa oficina, potencialmente interdisciplinar, foi elaborada pelos licenciandos participantes e desenvolvida com 21 estudantes do sexto ano de uma escola privada no município de Senhor do Bonfim. A referida oficina trabalhou os conteúdos de Astronomia - $\mathrm{O}$ sol e suas características, Movimentos de rotação e translação, Formação e constituição dos Planetas no sistema solar e Teoria de formação do universo. A abordagem interdisciplinar desse trabalho envolveu as disciplinas escolares Ciências, História e Geografia, "(...) demarcando assuntos como composição atmosférica, relevo e composição geológica, breve abordagem da formação do universo" (PLANO DE AULA DOS LICENCIANDOS). Algumas informações sobre a oficina estão dispostas no Quadro 1. 
Quadro 1 - Dados de identificação da oficina

\begin{tabular}{|c|c|c|c|c|}
\hline $\begin{array}{c}\text { Nome da } \\
\text { Oficina }\end{array}$ & $\begin{array}{c}\text { Disciplinas } \\
\text { escolares } \\
\text { envolvidas }\end{array}$ & Conteúdos & $\begin{array}{c}\text { Tema } \\
\text { transversal }\end{array}$ & Estratégia metodológica \\
\hline $\begin{array}{l}\text { O } \\
\text { aprendizado } \\
\text { das ciências } \\
\text { do céu por } \\
\text { meio do } \\
\text { sistema solar }\end{array}$ & $\begin{array}{l}\text { Ciências, } \\
\text { Geografia e } \\
\text { História. }\end{array}$ & $\begin{array}{l}\text { O sol e suas } \\
\text { caracteristicas, } \\
\text { Movimentos } \\
\text { de rotação e } \\
\text { translação, } \\
\text { Formação e } \\
\text { constituição } \\
\text { dos planetas e } \\
\text { Teoria de } \\
\text { formação do } \\
\text { universo. }\end{array}$ & Astronomia & $\begin{array}{l}\text { Caça palavras para sondar conhecimentos prévios } \\
\text { de astronomia, análise de imagem do sistema solar, } \\
\text { aula dialógica sobre Terra e Universo (modelos } \\
\text { astronômicos, história da astronomia), palestra } \\
\text { sobre caracteristicas dos planetas, resoluçåo em } \\
\text { grupo de atividade escrita, apresentação de trechos } \\
\text { do filme "Guardiões da galáxia" e posterior } \\
\text { produção textual identificando falácias no filme em } \\
\text { comparação com o sistema solar conhecido, } \\
\text { produção de modelo didático do sistema solar, } \\
\text { projeção de modelo do sistema solar por meio do } \\
\text { software Stellarium para comparação com os } \\
\text { modelos didáticos produzidos. }\end{array}$ \\
\hline
\end{tabular}

Fonte: Arquivos de Gisele Shaw.

A oficina pedagógica foi produzida no âmbito da disciplina Docência em Ciências: cultura escolar e cultura científica, que compõe o currículo do curso de Licenciatura em Ciências da Natureza da Univasf. Essa disciplina, de natureza teórico-prática, busca instrumentalizar os licenciandos acerca dos saberes da docência, da natureza da disciplina escolar Ciências e da interdisciplinaridade, que é inerente a essa natureza.

Assim, como parte das atividades da referida disciplina universitária, os licenciandos refletiram sobre a natureza interdisciplinar da disciplina Ciências, experienciando práticas interdisciplinares por meio de uma oficina pedagógica, pela qual poderiam construir conhecimentos e habilidades interdisciplinares. Nesse exercício, a reflexão na ação e sobre a ação, conforme Schön (2000), foi colocada como estratégia basilar à construção dessa formação interdisciplinar.

Essa investigação compôs uma pesquisa maior, do tipo Pesquisação, que buscou compreender as contribuições da pesquisa no ensino para a formação interdisciplinar de licenciados em Ciências da Natureza (SHAW; ROCHA, 2017; SHAW; FOLMER; ROCHA, 2017). O grupo de licenciandos que participou da turma de Docência em Ciências fez parte do segundo grupo de participantes da Pesquisação, iniciada no início do ano de 2014.

Os três licenciandos participantes da Oficina de que trata este artigo, foram denominados pelos pseudônimos Ana, Beth e Paulo, e possuíam, na época de coleta de dados, entre 19 e 26 anos de idade. Os dados referentes às concepções e ações dos mesmos foram coletados por meio de: questionário aplicado antes e depois da oficina (sondada sua concepção de interdisciplinaridade, sobre se a disciplina Ciências possui natureza interdisciplinar e se acha possível ensinar Ciências de modo interdisciplinar); formulário de planejamento da oficina; projeto investigativo da oficina; planos de aula (contemplando os dois dias de desenvolvimento da oficina, sendo um na escola e outro na universidade); anotações da professora com relato verbal da experiência; relatório da experiência na forma de artigo; formulário de autoavaliação dos licenciandos aplicado após a oficina; e entrevista (concedida por Beth). 
A pesquisa seguiu as normas prescritas pela Resolução no 466/12 do Conselho Nacional de Saúde, que trata da Ética em Pesquisa com Seres Humanos. A investigação, da qual resultou esse artigo, "A pesquisa como estratégia formativa de professores de Ciências para o trabalho interdisciplinar e por meio de temas transversais", foi aprovada pelo Comitê de Ética e Deontologia em Estudos e Pesquisas (Cedep) da Universidade Federal do Vale do São Francisco (Univasf), ad referendum, no dia 31 de março de 2014. Os dados foram coletados e analisados mediante assinatura de termos de compromisso dos participantes. A escola onde foi desenvolvida parte da oficina assinou termo de anuência de realização da pesquisa e foram seguidos todos os trâmites para resguardar a integridade dos participantes e sigilo de suas identidades.

Os dados coletados foram analisados mediante o método de Análise textual discursiva (MORAES, 2003; MORAES; GALIAZZI, 2006). Assim, após a coleta, esses dados foram organizados mediante transcrição e compilação em arquivo de texto e, depois, unitarizados, momento em que foram fragmentados em unidades, fundamentais ao estudo. Depois, cada uma dessas unidades foi reescrita de modo a ter sentido completo, e codificadas, conforme origem de fonte de dados e ideia principal envolvida.

Ao final da análise, as unidades de sentido foram divididas em dois grupos - conhecimentos e habilidades interdisciplinares -, que compõem as categorias pré-selecionadas no estudo. Em seguida, as unidades categorizadas foram articuladas ao referencial teórico da pesquisa, o que resultou num metatexto, considerado um novo emergente resultante da interpretação dos dados.

As categorias pré-selecionadas - componentes dos grupos conhecimentos e habilidades interdisciplinares - fazem parte da base teórica que fundamentou a análise da oficina, a ideia do pensamento interdisciplinar, segundo Spelt et al. $(2009,2010)$. Conforme visto anteriormente, para esses autores o pensamento interdisciplinar é composto por determinados conhecimentos e habilidades, que foram verificados nos licenciandos no decorrer da oficina pedagógica (Quadro 2).

Quadro 2 - Categorias de análise da pesquisa

\begin{tabular}{|c|l|}
\hline Grupo & \multicolumn{1}{c|}{ Categorias } \\
\hline Conhecimentos & $\begin{array}{l}\text { Conhecimento das disciplinas } \\
\text { Conhecimento dos paradigmas disciplinares } \\
\text { Conhecimento da interdisciplinaridade }\end{array}$ \\
\hline Habilidades & $\begin{array}{l}\text { Habilidades de alta ordem cognitiva } \\
\text { Habilidades de comunicação }\end{array}$ \\
\hline
\end{tabular}

Fonte: Arquivos de Gisele Shaw, com base em SPELT et al. $(2009,2010)$.

Esses tipos de conhecimentos e habilidades identificados nas categorias elencadas serão mais bem detalhados no tópico a seguir. 


\section{RESULTADOS E DISCUSSÕES}

As categorias selecionadas neste estudo foram predeterminadas com base no esquema de Spelt et al. (2009) e divididas em dois grupos: conhecimentos e habilidades. O grupo conhecimentos é constituído pelas categorias conhecimento das disciplinas, conhecimento dos paradigmas disciplinares e conhecimento da interdisciplinaridade. Já o grupo habilidades é composto pelas categorias habilidades de alta ordem cognitiva e habilidades de comunicação.

\section{O Grupo Conhecimentos}

A categoria Conhecimento das disciplinas envolve a detenção dos conteúdos, métodos, linguagem e epistemologia das disciplinas envolvidas no processo de interdisciplinaridade. Ainda que o professor não seja especialista em todas elas, é preciso que tenha abertura ao aprendizado, à pesquisa, a novos conhecimentos. Implica assumir que exercer a interdisciplinaridade abrange não somente o conhecimento da disciplina na qual o docente é especialista, mas também a flexibilidade de conhecer novas disciplinas e negociar aproximações e limites necessários à integração disciplinar (SPELT et al., 2009).

A categoria Conhecimento dos paradigmas disciplinares foi reportada à ideia de paradigma de Thomas Kunh (1998). Para este autor, um paradigma é uma realização científica que, em determinado momento histórico, fornece problemas e soluções provisórias a uma comunidade científica. Assim, ter conhecimento de paradigmas disciplinares envolve o conhecimento de paradigmas disciplinares cientificamente aceitos nas áreas envolvidas no processo interdisciplinar.

A categoria Conhecimento da interdisciplinaridade implica o conhecimento sobre a interdisciplinaridade, ou seja, uma compreensão não simplista do que seja a interdisciplinaridade, os aspectos que a envolvem e sobre como exercê-la, ainda que não haja consenso ou definição da própria concepção de interdisciplinaridade, ou sequer uma pedagogia própria (KLEIN, 2012). Concebemos, porém, que algum conhecimento de interdisciplinaridade aceito pela comunidade científica das áreas de educação e ensino seja necessário para a prática da mesma. Isso significa, por exemplo, que quem pretende trabalhar o ensino de modo interdisciplinar precisa saber a diferença entre interdisciplinaridade, multidisciplinaridade e transdisciplinaridade (SPELT et al., 2009).

\section{O Grupo Habilidades}

Do grupo habilidades, a categoria Habilidades de alta ordem cognitiva implica, segundo Spelt et al. (2009), com base em Boix Mansilla e Duraising (2007), Ivanitskaya et al. (2002) e Woods (2007), "the necessary ability to search, identify, understand, critically appraise, connect, and integrate theories and methods of different disciplines and to apply the resulting cognitive advancement together with continuous evaluation" 6 ( $p$. 9). Ainda conforme Spelt et al. (2009), isso implica que também abrange a capacidade

\footnotetext{
${ }^{6}$ Tradução sugerida pela autora: “(...) a capacidade necessária para pesquisar, identificar, compreender, apreciar criticamente, conectar e integrar teorias e métodos de diferentes disciplinas e aplicar o resultado do avanço cognitivo, juntamente com a avaliação contínua".
} 
de modificar perspectivas disciplinares e utilizá-las adequadamente. Para Ivanitskaya et al. (2002), estudos interdisciplinares facilitam a aquisição de estruturas de pensamento mais complexas e profundas, porque essa é uma característica da competência interdisciplinar, que depende do estabelecimento de conexões entre teorias, métodos, abordagens e paradigmas. Assim, o ensino interdisciplinar ajuda no desenvolvimento dessas habilidades de ordem superior, tais como de metacognição e de pensamento crítico (IVANITSKAYA et al., 2002).

De acordo com Newell e Green (1982), estudantes de cursos interdisciplinares desenvolvem a capacidade de raciocínio dedutivo (pois aprendem a identificar pressupostos disciplinares sob uma teoria), raciocínio por analogia (necessário para identificar ideias de uma disciplina respondendo problemas de outra) e raciocínio sintético (indispensável no processo de integração disciplinar), que são habilidades superiores de pensamento.

A categoria Habilidades de comunicação envolve a capacidade de comunicar-se adequadamente, de apresentar suas contribuições disciplinares, mas também saber tratar do assunto por meio da linguagem de outras disciplinas, de negociar pressupostos com outros especialistas, de agenciar diferenças disciplinares e de apresentar o avanço cognitivo gerado (SPELT et al., 2009).

\section{Análise dos Dados}

No grupo conhecimentos foi analisada a categoria Conhecimentos disciplinares com base nos conhecimentos gerais de Astronomia e sobre o ensino de Astronomia apresentados pelos licenciandos investigados. Isso porque, conforme Shulman (1986), "Mere content knowledge is likely to be us useless pedagogically as content free-skill" (p. 8). Para esse autor, o conhecimento pedagógico da disciplina tem tanto valor quanto o conhecimento específico da matéria. Assim, compreende-se que o conhecimento disciplinar abrange tanto os assuntos específicos da matéria quanto os conteúdos pedagógicos de como ensiná-la (referentes à didática específica da disciplina).

Os conhecimentos gerais de Astronomia, a que nos referimos, contempla a compreensão dos conteúdos de Astronomia trabalhados em nível de séries finais do Ensino Fundamental, conforme orientações dos Parâmetros Curriculares (PCNs) de Ciências Naturais (BRASIL, 1998).

Já os conhecimentos acerca do ensino de astronomia envolvem conhecimentos da Didática específica, tais como planejamento, estratégias de ensino e avaliação da aprendizagem, voltados ao ensino da Astronomia para alunos das séries finais do Ensino Fundamental. Então, o conhecimento sobre o ensino de Astronomia abarca o conhecimento pedagógico de saber lidar com a turma, operacionalizar o planejamento e desenvolver estratégias conforme o contexto apresentado, além de avaliar os avanços dos alunos, auxiliando-os no processo de ensino e aprendizagem em astronomia.

\footnotetext{
${ }_{7}^{7}$ Tradução sugerida pela autora: "O mero conhecimento do conteúdo é provavelmente tão pedagogicamente inútil quanto uma habilidade livre de conteúdo".
} 
Apesar de não ter sido aplicado um questionário ou atividade de sondagem dos conhecimentos específicos de Astronomia aos licenciandos, as explicações deles foram verificadas em fontes disponíveis. De forma geral, o grupo apresentou conhecimentos específicos de modo satisfatório, em conformidade a indicações dos PCNs de Ciências Naturais para séries finais do Ensino Fundamental, tais como modelos de elementos do universo: "Para organizar os elementos que os alunos incorporam para a transformação de seus modelos, um instrumento simples e eficaz é solicitar que desenhem representações do Universo, onde a Terra esteja presente, por várias vezes, durante esses estudos" (BRASIL, 1998, p. 64).

Beth demonstrou conhecimento sobre a composição química da atmosfera dos planetas. Ela se mostrou surpresa, por exemplo, ao relatar que os alunos pensavam que o ar que respiramos fosse constituído unicamente pelo gás oxigênio. A mesma explicou que expôs aos estudantes acerca da presença de outros gases e substâncias na atmosfera terrestre e, desse modo, os levou a comparar a atmosfera de outros planetas à do Planeta Terra. Beth observou que os estudantes não sabiam que no Planeta Marte havia vapor de água. Além de explicar como apresentou a ideia de vapor de água em Marte, ela também mencionou que trabalhou outras questões, tais como a História da organização do Universo e sobre os protagonistas envolvidos.

A licencianda Beth também explicou como trabalhou as ideias de translação e de rotação, associando o movimento de translação da Terra à periodicidade com que estudantes completam aniversário, além de mostrar as diferenças desses movimentos em outros planetas. Ela explicou que foi responsável por sugerir o desafio de perguntas e respostas. Conforme Miranda et al. (2016), o uso de jogos estimula os estudantes à aprendizagem de conteúdos de Astronomia, pois fortalece o processo de ensino e aprendizagem de modo divertido e prazeroso. Além do mais, Beth incentivou a participação dos alunos por meio de questionamentos, tais como sobre a composição dos planetas, e se os alunos achavam que em cada planeta poderiam respirar livremente. Para a mesma, era importante fomentar a participação dos estudantes na oficina.

Beth, entretanto, utilizou o termo "transmitir conhecimento" para apresentar o modo como expôs os conteúdos, apesar de demonstrar ter se preocupado em desenvolver estratégias didáticas divertidas: "Creio que pude transmitir bem o assunto, pois o conteúdo apresentado já foi trabalhado por nós, alunos, na universidade, e consegui transmiti-lo de forma interdisciplinar e bem divertida também" (BETH, QUESTIONÁRIO DE AVALIAÇÃO DA OFICINA). Mesmo assim, Beth criticou o modo conteudista como os conteúdos de ciências têm sido trabalhados nas escolas e afirmou a importância de associar os assuntos às vivências dos estudantes.

Paulo também relatou, durante a apresentação da experiência na universidade, que muitos alunos não sabiam da atual classificação de Plutão: a de não mais ser considerado planeta. Ele também apontou as limitações do sistema solar construído pelos alunos e como os mesmos as identificaram, tais como as diferenças entre as cores utilizadas nos modelos construídos e aquelas apresentadas no software Strellarium e, também, diferenças relacionadas aos anéis de Júpiter. Carvalho et al. (2016) e Sampaio e Rodrigues (2015) indicaram que o software Strellarium pode aumentar o interesse de estudantes em aprender conteúdos de Astronomia. De acordo com Carvalho et al. 
(2016), a utilização de recursos audiovisuais e dinâmicos no ensino, como é o caso dos simuladores, pode promover uma aprendizagem mais efetiva de fenômenos físicos, posto que os simuladores propiciam uma maior interação dos estudantes com a dinâmica do fenômeno modelado.

Quanto a seus conhecimentos pedagógicos, Paulo demonstrou valorizar a colaboração entre os participantes do grupo para garantir o sucesso da oficina. Segundo Santana (2015), a própria ação interdisciplinar pode favorecer a cooperação no grupo, mas é necessário primeiro se permitir ser interdisciplinar, ou seja, depende do desejo colaborativo. Paulo afirmou ainda que fomentou atividades de natureza investigativa, propiciando a reflexão dos alunos, incentivando a pesquisa e a resolução de dúvidas por meio do diálogo com eles. Além disso, Paulo mencionou que apresentou um filme com cenário do espaço, de modo a proporcionar uma atividade lúdica aos estudantes.

Ana relatou as dificuldades que os alunos encontraram em representar as manchas solares, mas ela não apresentou conhecimentos de conteúdos específicos nas fontes que investigamos. Não atribuímos esse fato à natureza tímida de seu comportamento, pois a análise não foi realizada apenas em fonte de dados de natureza verbal, tal como a apresentação da experiência em classe, mas, também, pela análise das respostas dadas no formulário de avaliação da oficina. Nesse formulário, Ana referiu-se aos conteúdos de Astronomia trabalhados com os estudantes, como "o assunto" ou "o conteúdo", sem mencionar especificamente quais foram. Consideramos que o conhecimento dela acerca do conteúdo poderia ter sido mais bem evidenciado caso houvessem mais dados a ser analisados (se a mesma, por exemplo, tivesse concedido entrevista).

Ana explicou ter participado de todas as etapas de realização do trabalho, desde o planejamento até a finalização do mesmo. Utilizou, no entanto, termos relativos a uma ideia transmissiva de ensino, tais como "manipulação do conteúdo" ou "exposição do conhecimento". Essa evidência de uma perspectiva transmissiva associada à Ana foi observada mesmo quando relatou acerca da participação dos alunos na oficina: "Favoreci de maneira a integrá-los na aula, eles falaram o que sabiam, perguntaram o que não sabiam" (ANA, QUESTIONÁRIO DE AVALIAÇÃO DA OFICINA). Apesar de considerar os conhecimentos prévios dos estudantes, esse tratamento do processo de ensino e aprendizagem de Ana demonstra o entendimento da construção do conhecimento como um processo simples, no qual o alcance de um conhecimento novo se dá pela obtenção de respostas prontas pelo professor.

Sobre a categoria Conhecimentos de paradigmas, observamos que os licenciandos demonstraram conhecer dois importantes paradigmas da astronomia: as Teorias Heliocêntrica e Geocêntrica. Também mencionaram os papéis de Galileu Galilei e Nicolau Copérnico, que trouxeram importantes contribuições à História da Astronomia.

Outro objetivo desse trabalho era discutir com os alunos brevemente o histórico da astronomia, conhecendo sobre grandes estudiosos dessa área, tais como Nicolau Copérnico e Galileu Galilei, apresentando a evolução do estudo e também modelos já utilizados de classificação do universo como Geocentrismo e Heliocentrismo (LICENCIANDOS, ARTIGO). 
Ainda no grupo Conhecimentos, quanto à categoria Conhecimento sobre a interdisciplinaridade, foi observado que houve avanços nas compreensões dos licenciandos no decorrer do processo. Tanto Ana quanto Beth, logo no início da disciplina Docência em ciências, demonstraram compreensões conceituais da interdisciplinaridade mais próximas da ideia de multidisciplinaridade.

Inicialmente, Beth confundiu interdisciplinaridade com multidisciplinaridade, afirmando que na interdisciplinaridade um tema é trabalhado por várias matérias. Depois, ela modificou essa percepção e mencionou o grande alcance dos conteúdos de Ciências, que podem ser trabalhados em várias matérias e facilitar a integração disciplinar. Ana também apresentou, inicialmente, sua concepção de interdisciplinaridade próxima ao conceito de multidisciplinaridade, mas, depois, ela indicou que um conteúdo de Ciências pode ser envolvido em discussões em várias disciplinas e, desse modo, facilitar a interdisciplinaridade. Logo, Ana e Beth alargaram seus conceitos de interdisciplinaridade, inicialmente confundindo a interdisciplinaridade com multidisciplinaridade e, depois, trazendo a ideia de que um único conteúdo pode envolver a integração de mais de uma disciplina. De acordo com Newell e Green (1982), a natureza interdisciplinar de uma questão é definida pelo seu escopo e pela necessidade da participação de mais de uma disciplina para poder respondê-la. Segundo Beth: "Ao dar uma aula sobre água é possível abordar diferentes disciplinas, desde a Química com os processos de ciclos da água, e os tratamentos que esta recebe para consumo humano, até Geografia, com as diferentes estruturas geográficas ocasionadas pela água" (BETH, QUESTIONÁRIO DE INTERDISCIPLINARIDADE PÓS-TESTE). Ou seja, Beth indicou que o alcance da abordagem do tema água envolve a contribuição de diversas disciplinas.

É importante considerar que, ainda que o processo de interdisciplinaridade envolva a integração disciplinar, as contribuições disciplinares não requerem o aprofundamento em cada matéria, e sim no tema comum. De acordo com Ivanitskaya et al. (2002), a abordagem interdisciplinar de ensino difere da tradicional, porque foge da necessidade de apostar num alto grau de aprofundamento em uma disciplina: ela não se preocupa em estimular a memorização de conceitos, mas substitui essa memorização pela concentração num tema central, pela aplicação de conhecimentos relativos a esse tema e pela reflexão sobre o processo de pensamento interdisciplinar (sobre as modificações disciplinares trazidas pelo processo). Apesar, porém, de considerarem as contribuições disciplinares, as licenciandas não evidenciaram compreender a importância da cooperação entre especialistas no processo de ensino interdisciplinar ou nas mudanças disciplinares ocorridas na experiência.

Assim, percebemos que, mesmo que as novas concepções mais alargadas de interdisciplinaridade trazidas por Beth e Ana não sejam as menos simplistas, elas continuam simplistas, uma vez que desconsideram a importância da troca coletiva de saberes e a reflexão sobre o enriquecimento das disciplinas com o acontecimento da prática interdisciplinar.

Paulo, apesar de não ter respondido os questionários sobre interdisciplinaridade, mencionou, no questionário avaliativo da oficina, que propiciou aos estudantes o envolvimento com atividades investigativas, “(...) que proporcionassem aos alunos encontrarem e refletirem sobre alguns conteúdos das áreas de Ciências, Geografia e His- 
tória" (PAULO, QUESTIONÁRIO AVALIATIVO DA OFICINA). Para ele, com a oficina os alunos se desenvolveram quanto à aquisição de conhecimentos relativos a características geográficas, históricas e científicas. Então, Paulo nos deu evidência de que entende que o trabalho interdisciplinar envolve a relação entre disciplinas, a partir da resolução de um mesmo problema, tema ou assunto.

Apesar, portanto, de os três licenciandos não mencionarem especificamente acerca do crescimento das disciplinas propiciado pelo trabalho interdisciplinar, podemos evidenciar avanços em suas concepções iniciais de interdisciplinaridade.

Passando ao grupo Habilidades, analisamos a categoria Habilidades de alta ordem cognitiva e podemos afirmar que não foi possível aferir de modo específico e individualizado acerca da aquisição dessas habilidades a partir dos dados coletados. De modo geral, entretanto, podemos afirmar que os licenciandos conseguiram pesquisar, identificar e integrar, de modo harmônico, métodos (uso de vídeos, produção textual identificação e análise de modelos) e conteúdos das disciplinas escolares - Ciências (O sistema solar), Geografia (Composição e relevo dos planetas do sistema solar) e História (História da formação do universo, Origem dos nomes dos planetas do sistema solar).

Embora seja preciso considerar que os assuntos da Astronomia naturalmente apresentem uma natureza interdisciplinar (BARROS; ASSIS; LANGHI, 2016; CARVALHO, et al., 2016; LANGHI; NARDI, 2014), os licenciandos souberam relacionar as disciplinas envolvidas de modo harmônico, de maneira a integrá-las. É possível perceber que eles souberam lidar com a interdisciplinaridade por meio da exploração da complementaridade de conteúdos e métodos das disciplinas envolvidas no processo (NEWELL; GREEN, 1982). Eles exploraram, juntamente com os estudantes, sobre planetas e universo, que são assuntos da disciplina Ciências, mas lidando com os modos operatórios da Geografia, pela exploração geográfica e construção de modelos didáticos (que também é metodologia utilizada em Ciências) e da História, pela contextualização histórica.

Apesar, contudo, de conseguirem integrar disciplinas na oficina, os licenciandos não puderam identificar ou perceber os avanços decorrentes dessa integração. Ou seja, eles não souberam avaliar o enriquecimento de cada disciplina no decorrer do processo: $O$ que foi agregado às disciplinas Geografia, Ciências e História por meio da experiência? Isso é uma reflexão metacognitiva que faz parte do exercício interdisciplinar. De acordo Gourgey (1998), segundo Ivanitskaya et al. (2002),

Whereas cognitive strategies enable one to make progress - to build knowledge - metacognitive strategies enable one to monitor and improve one's progress - to evaluate understanding and apply knowledge to new situations. Thus metacognition is vital to cognitive effectiveness (p. 82). ${ }^{8}$

\footnotetext{
${ }^{8}$ Tradução sugerida: "Considerando que as estratégias cognitivas permitem que se faça progresso - para construir o conhecimento - estratégias metacognitivas permitem monitorar e melhorar o progresso de alguém - para avaliar a compreensão do conhecimento e aplicá-lo a novas situações. Assim, a metacognição é vital para a eficácia cognitiva “(p. 82).
} 
Para Ivanitskaya et al. (2002), os estudantes de programas interdisciplinares aprimoram sua capacidade metacognitiva por meio do esforço em refletir sobre o processamento de sua própria forma de pensar. Isso implica trabalho formativo focado na reflexão, reflexão na ação e reflexão sobre a ação e sobre a própria reflexão. Logo, é um trabalho de autoavaliação e autorreflexão constante (IVANITSKAYA et al., 2002).

Talvez os licenciandos não tenham de fato evidenciado o progresso disciplinar mediante as suas próprias limitações quanto à compreensão do que seja a prática interdisciplinar. Também é preciso considerar que, conforme Ivanitskaya et al. (2002), a conversão de disciplinas num tema, como é no caso a Astronomia, promove o desenvolvimento de habilidades por meio do esforço intelectual no próprio ato de integração, pela "(...) analysis, comparison, and contrast of perspectives contributed by each discipline" 9 (IVANITSKAYA et al., 2002, p. 101). É necessário ponderar que a prática interdisciplinar não é instantânea, mas requer tempo para ser desenvolvida, amadurecida (SPELT et al., 2009). Inclusive, de acordo com Ivanitskaya et al. (2002), a estrutura interdisciplinar de conhecimento é desenvolvida com o avanço da capacidade cognitiva de alta ordem "specifically, metacognitive skills, critical thinking, and personal epistemology" ${ }^{10}$ (p. 101).

É preciso, todavia, reconhecer os avanços relativos a esse sucesso da integração. Ainda, é necessário evidenciar que a utilização de modelos didáticos de planetas e a comparação dos mesmos com os planetas observados por meio do uso do software Strellarium, foi uma estratégia interessante para acompanhar o conhecimento obtido pela integração de contribuições trazidas pelas disciplinas Geografia e Ciências, de modo bem-sucedido. A Figura 1 traz um dos modelos construídos pelos estudantes durante a oficina.

Figura 1 - Modelo construído na oficina pelos estudantes

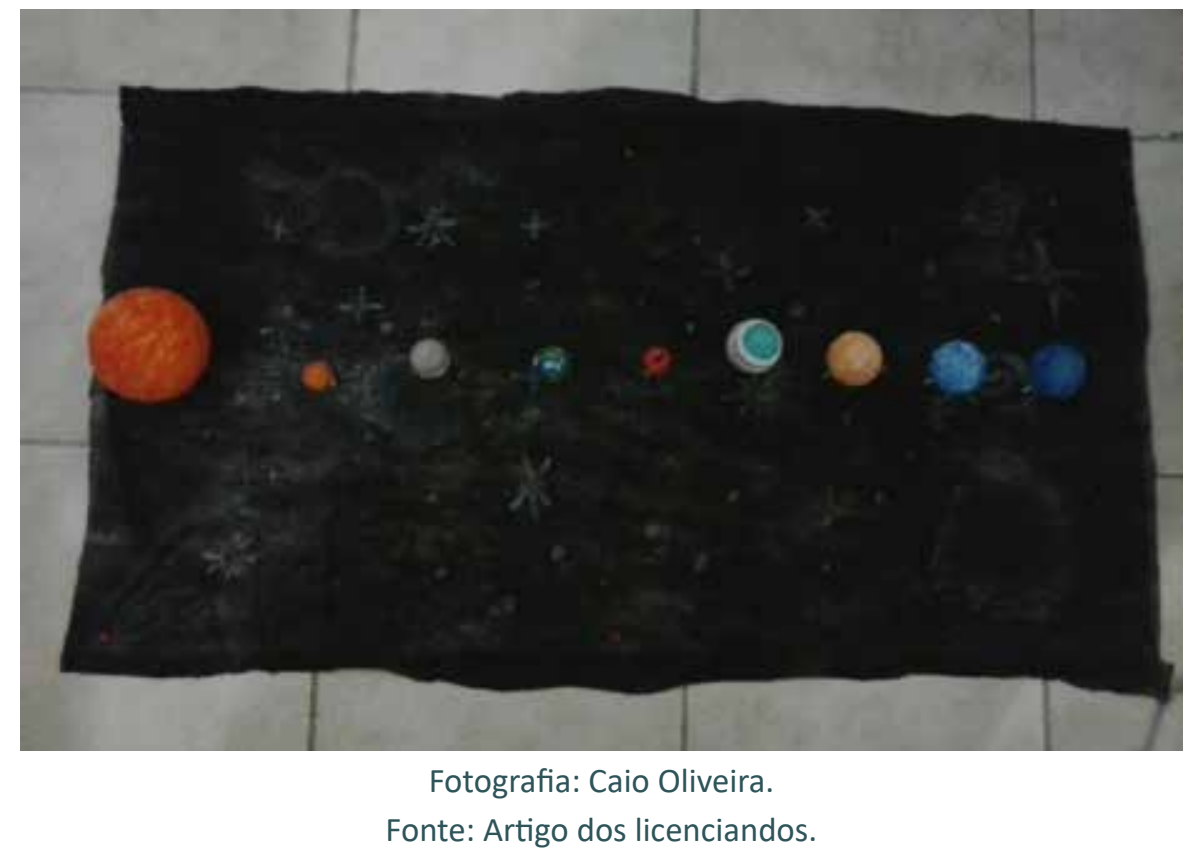

${ }^{9}$ Sugestão de tradução: “(...) análise, comparação e contraste de perspectivas dadas por cada disciplina”.
${ }^{10}$ Sugestão de tradução: "especificamente, habilidades metacognitivas, pensamento crítico e epistemologia pessoal”. 
Podemos afirmar que os licenciandos conseguiram organizar o conhecimento e articulá-lo de modo interessante e favorecedor da integração disciplinar. De acordo com Ivanitskaya et al. (2002), apesar de a organização estrutural do conhecimento não ser característica exclusiva da abordagem de ensino tradicional, a abordagem interdisciplinar promove a integração consistente do conhecimento trabalhado, por meio de uma estruturação mais complexa do conhecimento.

Sobre a categoria Habilidades de comunicação, verificamos que houve satisfatoriedade no desenvolvimento dos licenciandos. Esses avanços relacionam-se ao fato de que, apesar de não termos observado o desenvolvimento da oficina, os dados investigados nos deram algumas evidências. Verificamos que o grupo conseguiu apresentar os conteúdos de Astronomia de modo objetivo, contextualizado e fomentando a participação dos alunos a partir do inquérito. Os licenciandos sempre questionavam os estudantes sobre o que pensavam acerca do que estava sendo estudado, e elaboraram estratégias que propiciavam sua participação. Além disso, afirmaram ter desenvolvido aulas dialógicas com os estudantes, o que evidencia uma boa comunicação no processo de ensino e aprendizagem.

Foi observado, então, que os três licenciandos conseguiram estabelecer conexões dos conteúdos de Ciências (Sistema Solar) com assuntos da Geografia (Estrutura dos planetas) e da História (História do surgimento do universo), ao mesmo tempo em que utilizaram metodologias adequadas ao trabalho com ensino de Astronomia e História (uso de filme, simulador, pesquisa, modelagem).

\section{ALGUMAS CONSIDERAÇÕES}

É sabido que, apesar de a prática interdisciplinar ser fomentada tanto pela literatura especializada quanto pela legislação educacional, é preciso o alargamento do conhecimento de como fazê-lo. Concebemos a necessidade de viabilizar o desenvolvimento do pensamento interdisciplinar de estudantes de Licenciatura em Ciências, por meio da aquisição de uma formação interdisciplinar no Ensino Superior. Esse pensamento interdisciplinar, conforme Spelt et al. (2009), é complexo e constituído por conhecimentos e habilidades, além de condições específicas para tal.

Investigando três licenciandos em Ciências da Natureza, durante uma oficina interdisciplinar, verificamos o desenvolvimento de alguns dos conhecimentos e habilidades indicados por Spelt et al. (2009). Foi observado que os três licenciandos demonstraram ter conhecimentos acerca do ensino de Astronomia, e dois deles indicaram que também tinham conhecimentos específicos da Astronomia (prévios e adquiridos). Os três participantes também comprovaram ter conhecimentos de paradigmas da disciplina Ciências, mas ainda precisam alargar suas concepções de interdisciplinaridade, que pareceram estar mais próximas da ideia de integração. É preciso conceber, entretanto, que houve o desenvolvimento das concepções iniciais de interdisciplinaridade desses participantes no decorrer da oficina.

Sobre a aquisição de habilidades de alta ordem, foi observado que os licenciandos demonstraram habilidades relacionadas à pesquisa, à identificação e à integração disciplinar, mas não adquiriram a capacidade de identificação dos avanços das discipli- 
nas com o processo interdisciplinar, além da empregabilidade e da avaliação contínua desses avanços (SPELT, et al., 2009). Os três licenciandos demonstraram, porém, boa capacidade de comunicação com os estudantes.

A investigação em questão propiciou o conhecimento de bases importantes para a identificação da formação interdisciplinar no Ensino Superior e apontou conhecimentos e habilidades necessários nesse processo. No estudo apresentado, verificamos que não houve a constituição do pensamento interdisciplinar nos licenciandos, mas que esse pensamento estava em construção. Também foi possível identificar quais conhecimentos e habilidades estiveram ausentes para que isso ocorresse. Desse modo, é preciso prosseguir com estudos empíricos que validem as ideias de Spelt et al. (2009), os quais podem contribuir de modo significativo para a formação interdisciplinar de professores e com estudos sobre a interdisciplinaridade no ensino.

\section{AGRADECIMENTOS}

Agradeço a Geraldo Soares da Silva Junior, por auxiliar na transcrição da entrevista, e a Patrick Everton do Nascimento, por realizar a revisão do texto.

\section{REFERÊNCIAS}

BARROS, L. G.; ASSIS, A.; LANGHI, R. Proposta de construção de espectroscópio como alternativa para o ensino de Astronomia. Caderno Brasileiro de Ensino de Física, v. 33, n. 3, p. 1.026-1.046, dez. 2016.

BIGGS, J. B. Teaching for quality learning at university: what the student does. 2. ed. Buckingham: Open University Press, 2003.

BOIX MANSILLA, V.; DURAISING, E. D. Targeted assessment of students' interdisciplinary work: Na empirically grounded framework proposed. The Journal of Higher Education, v. 78, n. 2, 2007.

BRASIL. Secretaria de Educação Fundamental. Parâmetros curriculares nacionais: Ciências Naturais. Brasília: MEC; SEF, 1998.

CARVALHO, C. L. de; ZANITTI, M. H. R.; FELICIDADE, B. do L.; GOMES, A. D. T.; DIAS, E. W.; COELHO, F. O. Um estudo sobre o interesse e o contato de alunos do Ensino Médio com astronomia. Areté: Revista Amazônica de Ensino de Ciências, v. 9, n. 18, 2016.

DELIZOICOV, D.; ANGOTTI J. A.; PERNAMBUCO, M. M. Ensino de ciências: fundamentos e métodos. São Paulo: Cortez, 2002.

FAZENDA, I. C. A. Desafios e perspectivas do trabalho interdisciplinar no Ensino Fundamental. In: FAZENDA, I. C. (org.). Novos enfoques da pesquisa educacional. São Paulo: Cortez, 2010.

FAZENDA, I. C. A. Interdisciplinaridade: História, teoria e pesquisa. Campinas, SP: Papirus, 2012a.

FAZENDA, I. C. A. (org.). A aquisição de uma formação interdisciplinar de professores. In: FAZENDA, I. C. A. (org.). Didática e interdisciplinaridade. Campinas, SP: Papirus, 2012b.

GOURGEY, A. F. Metacognition in basic skills instruction. Instructional Science, 26,

p. 81-96, 1998.

IVANITSKAYA, L.; CLARK, D.; MONTGOMERY, G.; PRIMEAU, R. Interdisciplinary learning: Process and outcomes. Innovative Higher Education, v. 27, n. 2, 2002.

KLEIN, J. T. Ensino interdisciplinar: didática e teoria. In: FAZENDA, Ivani (org.). Didática e interdisciplinaridade. Campinas, SP: Papirus, 2012.

KUHN, T. A estrutura das revoluções científicas. 5. ed. São Paulo: Perspectiva, 1998.

LANGHI, R.; NARDI, R. Justificativas para o ensino de Astronomia: o que dizem os pesquisadores brasileiros? Revista Brasileira de Pesquisa em Educação em Ciências, v. 14, n. 3, 2014.

LENOIR, Y. Três interpretações da perspectiva interdisciplinar em educação em função de três tradições culturais distintas. Revista E-Curriculum, São Paulo: PUCSP, v. 1, n. 1, 2005.

MIRANDA, J. C.; GONZAGA, G. R.; COSTA, R. C.; FREITAS, C. C. C.; CÔRTES, K. C. Jogos didáticos para o ensino de Astronomia no Ensino Fundamental. Scientia Plena, v. 12, n. 2, 2016. 
MORAES, R. Uma tempestade de luz: a compreensão possibilitada pela análise textual discursiva. Ciência \& Educação, v. 9, n. 2, p. 191-211, 2003.

MORAES, R.; GALIAZZI, M. do C. Análise textual discursiva: processo reconstrutivo de múltiplas faces. Ciência \& Educação, v. 12, n. 1, p. 117-128, 2006.

MOREIRA, M. A. Metodologias de pesquisa em ensino. São Paulo: Editora Livraria da Física, 2011.

NEWELL, W. H.; GREEN, W. J. Defining and Teaching Interdisciplinary Studies. Improving College and University Teaching, v. 30, n. 1, p. 23-30, winter 1982.

SAMPAIO, T. A. de S. M.; RODRIGUES, E. da S. Método didático para o ensino de astronomia: utilização do software Stellarium em conjunto com aulas expositivas no ensino médio. Revista Ciência e Desenvolvimento, v. 8, n. 2, 2015.

SANTANA, Elisangela Barreto. Abordagem CTS no ensino de astronomia: formação de professores mediada pela situação problema "Centro de Lançamento de Alcântara". 2015. 126f. Dissertação (Mestrado) Universidade Federal do Pará, Instituto de Educação Matemática e Científica, , Belém, 2015.

SCHON, D. A. Educando o profissional reflexivo: um novo design para o ensino e a aprendizagem. Trad. Roberto Cataldo Costa. Porto Alegre: Artmed, 2000.

SHAW, G. S. L.; ROCHA, J. B. T. Tentativa de construção de uma prática docente interdisciplinar em ciências. Experiências em Ensino de Ciências, v. 12, n. 1, 2017.

SHAW, G. S. L.; FOLMER, V.; ROCHA, J. B. T. Uma revisão sobre a interdisciplinaridade no ensino e a formação de professores. Revista Ciências \& Ideias, v. 8, n. 1, jan./abr. 2017.

SHULMAN, L. S. Those who understand: Knowledge growth in teaching. Educational Researcher, v. 15, n. 2, Feb, 4-14, 1986 (AERA Presidential Address). Available from: http://links.jstor.org/sici?sici=0013-189X\% 28198602\%2915\%3A2\%3C4\%3ATWUKGI\%3E2.0.CO\%3B2-X. Cited: 15 maio 2018.

SPELT, E. J. H.; BIEMANS, H. J. A.; TOBI, H.; LUNING, P. A.; MULDER, M. Teaching and Learning in Interdisciplinary Higher Education: A Systematic Review. Educ. Psychol. Rev., 21, nov. 2009.

SPELT, E. J. H.; BIEMANS, H. J. A.; LUNING, P. A.; TOBI, H.; MULDER, M. Interdisciplinary thinking in agricultural and life sciences higher education. Comm. Appl. Biol. Sci, Ghent University, v. 75, n. 1, 2010.

WOODS, C. Researching and developing interdisciplinary teaching: Towards a conceptual framework for classroom communication. Higher Education, v. 54, n. 6, 2007. 